\title{
Advanced magnetic visualization of Mt. Vesuvius shallow plumbing system by probability tomography
}

\author{
Teresa Iuliano $\left({ }^{1}\right)$, Paolo Mauriello $\left({ }^{2}\right)$ and Domenico Patella $\left({ }^{1}\right)$ \\ (1) Dipartimento di Scienze Fisiche, Università «Federico II», Napoli, Italy \\ (2) Istituto per le Tecnologie Applicate ai Beni Culturali, CNR, Roma, Italy
}

\begin{abstract}
This paper reports the results of the application of a new magnetic probability tomography imaging method to the aeromagnetic data set collected by AGIP on Mt. Vesuvius in 1978. A magnetic dipole occurrence probability function is defined, in order to contain all information about the whole class of equivalent sources compatible with the original survey data set. An advanced 3D visual system is also applied for the first time to visualise the spacial pattern of the magnetic dipole occurrence probability function. It is shown that in the case of Vesuvius, the shallow plumbing system is characterised by a high probability of occurrence of a dipole at $2 \mathrm{~km}$ of depth b.s.l., nearly directed along the present main earth's magnetic field. This leads to hypothesize that the top terminal portion of the volcanic apparatus is completely filled with magnetised volcanic material.
\end{abstract}

Key words probability tomography - magnetic anomaly - Vesuvius volcano

\section{Introduction}

Probability tomography was first introduced to image self-potential anomaly sources (Patella, 1997a,b) and then extended to analyse geoelectrical (Mauriello et al., 1998; Mauriello and Patella, 1999a), natural electromagnetic induction (Mauriello and Patella, 1999b, 2000), gravity (Mauriello and Patella, 2001a,b) and magnetic data (Mauriello and Patella, 2001c). It is an interpretation tool,

Mailing address: Prof. Domenico Patella, Dipartimento di Scienze Fisiche, Università «Federico II», Complesso Universitario Monte S. Angelo, Via Cintia, 80126 Napoli, Italy; e-mail: patella@na.infn.it which accounts for the inherently uncertain nature of the geophysical inversion problem, due to the many different source configurations that can be modelled compatibly with a given data set.

Probability tomography has been already applied to the analysis of self-potential, geoelectrical, gravity and magnetic data sets collected in the volcanic area of Mt. Vesuvius (Naples, Italy) (Di Maio et al., 1998; Patella and Mauriello, 1999; Iuliano et al., 2001). The purpose of this paper is to repropose the to-mographic imaging of the magnetic sources beneath Mt. Vesuvius, using a new formulation of the magnetic probability tomography theory (Mauriello and Patella, 2001c) coupled with a 3D object-oriented Advanced Visual System (AVS).

In the following sections, the new magnetic probability tomography imaging method and the most important aspects of a 3D AVS visual development tool are described. Then 
the results of the application to the Vesuvius aeromagnetic data set collected by Cassano and La Torre (1987) are presented and discussed.

\section{Probability tomography theory}

\subsection{The 3D magnetic occurrence probability} function

We assume a coordinate system with the $(x, y)$ plane at sea level and the $z$-axis positive upwards. Let $\boldsymbol{B}(\boldsymbol{r})$ be a static magnetic induction field, evaluated at a grid of points $r \in S$, where $S$ is a portion of the earth's surface characterized by a topography function $z(x, y)$. In terms of total magnetization vector $\boldsymbol{M}(\boldsymbol{r}), \boldsymbol{B}(\boldsymbol{r})$ can be expressed as (Jackson, 1998; Mauriello and Patella, 2001c)

$$
\boldsymbol{B}(\boldsymbol{r})=\frac{\mu_{0}}{4 \pi} \int_{V} \frac{3 \boldsymbol{n}\left[\boldsymbol{n} \cdot \boldsymbol{M}\left(\boldsymbol{r}^{\prime}\right)\right]-\boldsymbol{M}\left(\boldsymbol{r}^{\prime}\right)}{\left|\boldsymbol{r}-\boldsymbol{r}^{\prime}\right|^{3}} d V
$$

where $V$ is a volume containing all the magnetic sources at $\boldsymbol{r}^{\prime} \in V$ and $\boldsymbol{n}$ is the unit vector in the direction of $\boldsymbol{r}-\boldsymbol{r}^{\prime}$.

Assuming the magnetic field on the earth's surface as due to a finite distribution of $Q$ elementary magnetic sources, eq. (2.1) can be discretized as follows:

$$
\boldsymbol{B}(\boldsymbol{r})=\sum_{q=1}^{Q} \frac{3 \boldsymbol{n}_{\boldsymbol{q}}\left(\boldsymbol{n}_{\boldsymbol{q}} \cdot \boldsymbol{d}_{\boldsymbol{q}}\right)-\boldsymbol{d}_{\boldsymbol{q}}}{\left|\boldsymbol{r}-\boldsymbol{r}_{\boldsymbol{q}}\right|^{3}}
$$

where $\boldsymbol{n}_{q}$ is the unit vector in the direction $\boldsymbol{r}-\boldsymbol{r}$ and the $q$-th generic element is a small volume $\Delta V_{q}$ centred at $\boldsymbol{r}_{q}$ with magnetization $\boldsymbol{M}\left(\boldsymbol{r}_{q}\right)$ and magnetic moment $\boldsymbol{d}_{q}$ given by

$$
\boldsymbol{d}_{q}=\frac{\mu_{0}}{4 \pi} \boldsymbol{M}\left(\boldsymbol{r}_{q}\right) d V_{q}
$$

Using the principles of the probability tomo- graphy (Patella, 1997a,b), at first the total power $\Lambda_{u}$, associated with the component $B_{u}(\boldsymbol{r})$ of $\boldsymbol{B}(\boldsymbol{r})$ along a generic direction identified by the unit vector $\boldsymbol{u}$, is defined as

$$
\Lambda_{u}=\int B_{u}^{2}(\boldsymbol{r}) d S=
$$

$(S)$

$$
=\sum_{q=1}^{Q}\left[\sum_{v=x, y, z} d_{q v} \int_{(S)} B_{u}(\boldsymbol{r}) \Re_{u v}\left(\boldsymbol{r}-\boldsymbol{r}_{\boldsymbol{q}}\right) d S\right]
$$

where the explicit expressions of the three $\mathfrak{R}_{u v}\left(\boldsymbol{r}-\boldsymbol{r}_{q}\right)$ functions $(v=x, y, z)$ are given by

$$
\begin{gathered}
\Re_{u x}\left(\boldsymbol{r}-\boldsymbol{r}_{q}\right)= \\
=\frac{1}{\left|\boldsymbol{r}-\boldsymbol{r}_{\boldsymbol{q}}\right|^{3}}\left[3 n_{q x}\left(n_{q x} \boldsymbol{i} \cdot \boldsymbol{u}+n_{q y} \boldsymbol{j} \cdot \boldsymbol{u}+n_{q z} \boldsymbol{k} \cdot \boldsymbol{u}\right)-\boldsymbol{i} \cdot \boldsymbol{u}\right] \\
\Re_{u y}\left(\boldsymbol{r}-\boldsymbol{r}_{\boldsymbol{q}}\right)=
\end{gathered}
$$$$
=\frac{1}{\left|\boldsymbol{r}-\boldsymbol{r}_{\boldsymbol{q}}\right|^{3}}\left[3 n_{q y}\left(n_{q x} \boldsymbol{i} \cdot \boldsymbol{u}+n_{q y} \boldsymbol{j} \cdot \boldsymbol{u}+n_{q z} \boldsymbol{k} \cdot \boldsymbol{u}\right)-\boldsymbol{j} \cdot \boldsymbol{u}\right]
$$$$
\Re_{u z}\left(\boldsymbol{r}-\boldsymbol{r}_{q}\right)=
$$$$
=\frac{1}{\left|\boldsymbol{r}-\boldsymbol{r}_{\boldsymbol{q}}\right|^{3}}\left[3 n_{q z}\left(n_{q x} \boldsymbol{i} \cdot \boldsymbol{u}+n_{q y} \boldsymbol{j} \cdot \boldsymbol{u}+n_{q z} \boldsymbol{k} \cdot \boldsymbol{u}\right)-\boldsymbol{k} \cdot \boldsymbol{u}\right]
$$

with $n_{q v}(v=x, y, z)$ being the components of the unit vector $\boldsymbol{n}_{q}$.

Then, a three-component Magnetic Occur- 
rence Probability (MOP) function $\eta_{u v}^{(M)}\left(\boldsymbol{r}_{q}\right)$ is defined as (Mauriello and Patella, 2001c)

$$
\begin{gathered}
\eta_{u v}^{(M)}\left(\boldsymbol{r}_{q}\right)= \\
=C_{u v}^{(M)} \int_{-X-Y}^{X} \int_{(v=x, y, z),}^{Y} B_{u}(\boldsymbol{r}) \Re_{u v}\left(\boldsymbol{r}-\boldsymbol{r}_{\boldsymbol{q}}\right) g(z) d x d y, \\
(v+6)
\end{gathered}
$$

where

$$
\begin{gathered}
C_{u v}^{(M)}= \\
=\left[\int_{-X-Y}^{X} \int_{u}^{Y} B_{u}^{2}(\boldsymbol{r}) g(z) d x d y \int_{-X-Y}^{X} \int_{\Re^{\prime}}^{Y} \Re_{u v}^{2}\left(\boldsymbol{r}-\boldsymbol{r}_{q}\right) g(z) d x d y\right]^{-1 / 2}, \\
(v=x, y, z),
\end{gathered}
$$

and $g(z)$, called the topographic surface regularization factor, is

$$
g(z)=\sqrt{1+(\partial z / \partial x)^{2}+(\partial z / \partial y)^{2}} .
$$

Each $\eta_{u v}^{(M)}$ function satisfies the bounding condition $-1 \leq \eta_{u v}^{(M)}\left(\boldsymbol{r}_{q}\right) \leq+1$ and three $\eta_{u v}^{(M)}$ values $(v=x, y, z)$ can be computed at each $\boldsymbol{r}_{q}$. Each value is interpreted as the probability, with which the homologous component $M_{v}(v=x, y, z)$ of a $\boldsymbol{M}$-field can at $\boldsymbol{r}$ be considered responsible for the measured component for the magnetic field.

In geophysical exploration, either the zcomponent or the modulus of the earth's magnetic field is usually measured and a scalar secondary field (the anomalous field) is evaluated in order to identify local sources of magnetic anomaly. In the case of the so-called total field survey, the scalar anomalous field is obtained by subtracting from the measured modulus of the earth's magnetic field the modulus of the known primary magnetic field. Since the secondary field is always a very small fraction of the primary field, it can be readily shown that in the case of a total field survey the scalar secondary field is the projection of the secondary vector field along the direction of the primary vector field (Blakely, 1996). This direction can generally be assumed uniform within the areas normally considered in geophysical prospecting (Parasnis, 1997). Thus, from now on we will consider $\boldsymbol{B}(\boldsymbol{r})$ as the secondary vector field, and the scalar component of $\boldsymbol{B}(\boldsymbol{r})$ along any fixed direction as the object of analysis in the new tomographic approach.

\section{3D probability tomography procedure}

The 3D tomography procedure for imaging the sources of a magnetic field, measured on a generally non-flat topography, consists in a reiterated computation code, involving the scanner functions $\Re_{u v}$ and the $B_{u}(\boldsymbol{r})$ field data set.

In practice, since we do not know the position of the real sources generating the anomalous magnetic field, we use a synthetic source of unitary strength to scan the $x, y, z$ half-space below the survey area (tomospace), in order to search where the real sources can be located in probabilistic sense. The scanning operation is performed by computing the crosscorrelation integrals in eq. (2.6) for each point $\left(x_{q}, y_{q}, z_{q}\right)$ of a regular grid within the tomospace. At each point, the value of each integral is interpreted as the probability of occurrence of the relative magnetic source component, whose positive or negative orientation depends on whether it is $\eta>0$ or $\eta<0$. By scanning the tomospace, using, e.g., a sequence of horizontal slices spaced from each other by a constant depth interval, we can finally obtain a 3D image of the equivalent magnetic source distribution underground in a probabilistic sense.

In order to improve the filtering property of the scanning procedure, for each $\boldsymbol{r}_{q}$ of the tomospace it is advisable to use varying sizes of the integration surface in eq. (2.6). The smallest size is the $[-X, X] \times[-Y, Y]$ domain fully 
containing the surface trace of the magnetic response of the scanning source element placed at $\boldsymbol{r}_{q}$. The greatest surface is, of course, the largest rectangle fitting to the whole survey area. The highest $\left|\eta\left(\boldsymbol{r}_{q}\right)\right|$ is taken with its sign as the most appropriate source occurrence probability at $\boldsymbol{r}_{q}$.

\section{Advanced data visualization}

In the most general term, data visualization is the process of representing data as a graphic display on a computer screen. In practice, data visualization gives an insight into data allowing the interpreter to graphically view structure and changes in the data. Generally speaking, there are two things the interpreter wants to do with data: analyse it to obtain information and present it to others to share that information. In either case, an effective visualization is an important tool. An Advanced Visual System (AVS) extends the obvious advantages of standard visualization by sophisticated 3D representations of data to be developed.

Modern 3D AVS softwares are considered object-oriented, visual development tools, enabling one to build reusable application components and complex visualization applications. AVS softwares become very useful when they can support different object-oriented techniques, such as encapsulation of data and methods, class inheritance, templates and instances, object hierarchies and polymorphism. Very shortly, a 3D AVS tool can be considered a visual development environment that can be used to connect, define, assemble and manipulate objects and related applications through mousedriven operations. The objects and applications that are connected and assembled control how data is processed and how it is displayed. The interpreter can even add a user interface to create a complete application that can be delivered as a stand-alone application.

We have applied a 3D AVS software (Advanced Visual Systems Inc., 1998) to visualize the results of the application of the magnetic probability tomography to the volcanic area of Mt. Vesuvius. Of course, as we cannot reproduce all the dynamical capabilities of the 3D AVS, we show in the following section only a particular perspective of the tomographic images from the most effective point of view.

\section{Mt. Vesuvius magnetic tomography and AVS imaging}

Vesuvius is considered one of the most risky active volcanoes in the world, because of its volcanic history and closeness to the city of Naples, Italy. The results achieved so far (Iuliano et al., 2001, and references therein) indicate that the shallow part of the volcano is made of a unique central plumbing system, entirely filled of altered volcanics in the summit portion. This peculiarity makes the volcano extremely hazardous, since explosion becomes a highly probable event in case of renewal of extruding activity. The magnetic study can notably help to ascertain whether such a condition really occurs. To this purpose, we show the results of the application of the probability tomography to the aeromagnetic total field data set collected in 1978 by the Italian Oil Company AGIP to study the volcano-geothermal structure of Mt. Vesuvius. A cesium optical pumping magnetometer was used at the constant altitude of $1460 \mathrm{~m}$ a.s.l.

Figure 1 shows the original total magnetic field map (after Cassano and La Torre, 1987). Subtracting a constant regional field in the survey area with intensity $30100 \mathrm{nT}$, inclination $56.30^{\circ}$ and declination $-1^{\circ}$, the residual effect is a large and intense positive anomaly with peak value of approximately $1800 \mathrm{nT}$, located in the central part of the volcanic system.

The 3D AVS pictures in fig. 2a-c show the pattern of the MOP functions $\eta_{u x}^{(M)}, \eta_{u y}^{(M)}$ and $\eta_{u z}^{(M)}$, respectively, drawn by plotting the MOP isosurface of the lower limit of the coloured palette corresponding to the decimal step where the highest absolute MOP values occur. Only absolute MOP values equal or greater than 0.6 have been considered in fig. 2a-c. In particular, fig. $2 \mathrm{c}$ shows a well defined nucleus centred at about $2 \mathrm{~km}$ b.s.l., including the lowest computed values of $\eta_{u z}^{(M)}$, falling in the range from -0.9 to -1 . Accordingly, fig. 2 b shows a smaller nucleus, centred again at about $2 \mathrm{~km}$ b.s.l., including the highest computed values of $\eta_{u v}^{(M)}$, falling in the 


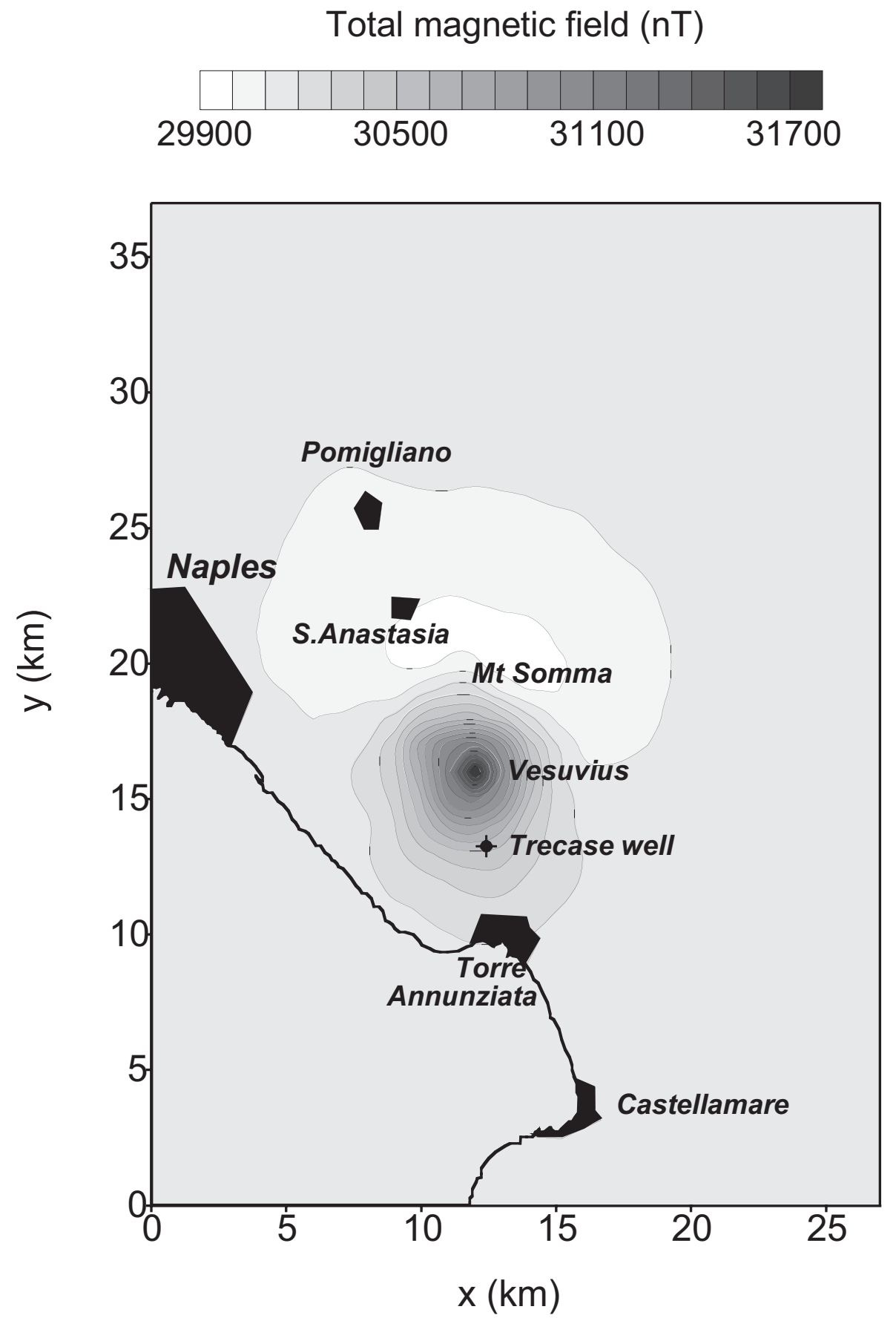

Fig. 1. The total field aeromagnetic map in the volcanic area of Mt. Vesuvius (after Cassano and La Torre, 1987). 


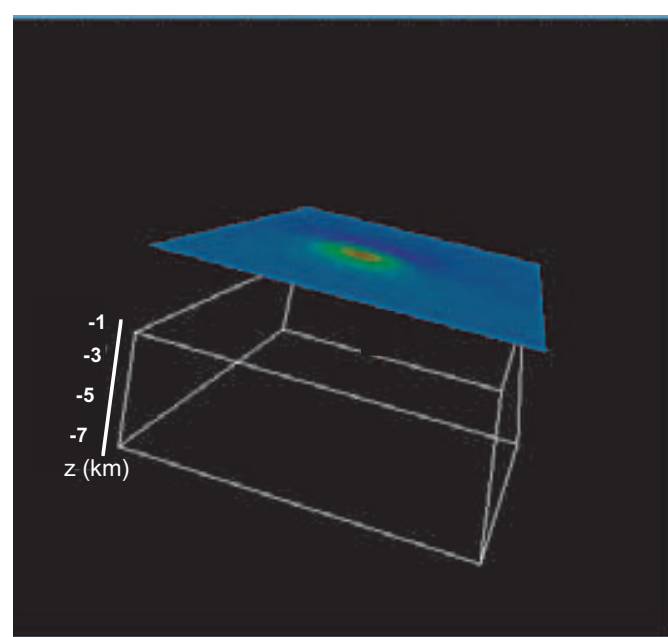

(a)

$$
\eta_{u x}^{(M)} \geq 0.6
$$

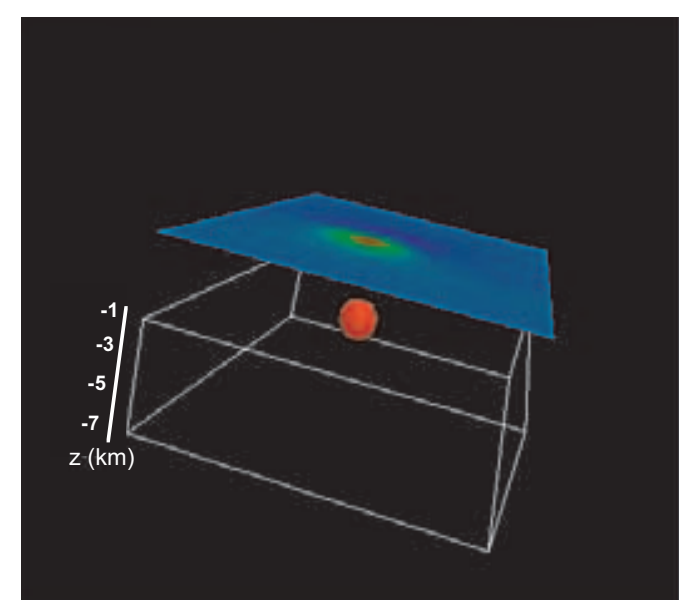

(b)

$$
0.6 \leq \eta_{u y}^{(M)} \leq 0.7
$$

range from 0.6 to 0.7 . No signal above the threshold of 0.6 is present in the $\eta_{u x}^{(M)}$ tomography of fig. 2a. Combining all these features, the relevant information is the high occurence probability of an inclined dipole centred at $x=$ $12 \mathrm{~km}, y=17 \mathrm{~km}$ and $z=-2 \mathrm{~km}$, with dominant

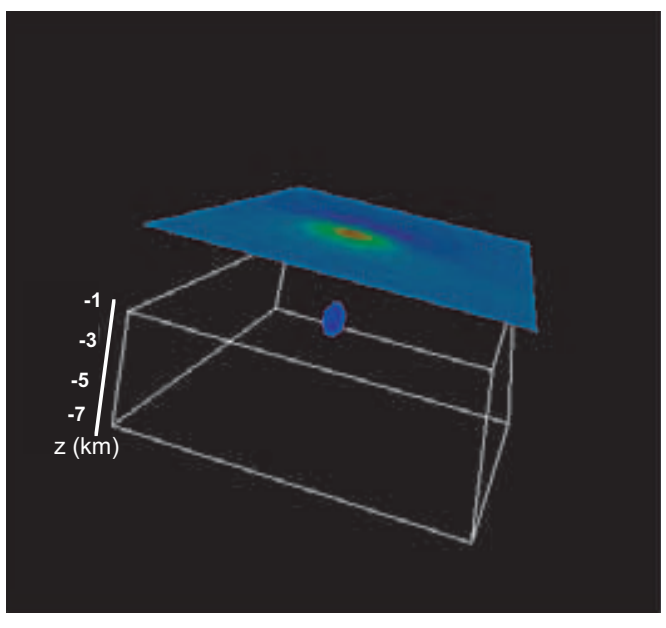

(C)

$$
-1 \leq \eta_{u z}^{(M)} \leq-0.9
$$

Fig. 2a-c. 3D AVS imaging of the magnetization occurrence probability function along the $x$-axis (a); $y$-axis (b) and $z$-axis (c). The top slice is the experimental map of the residual total magnetic field.

negative vertical component and positive horizontal component directed along the $y$-axis.

Figure 3a,b shows the comparison between the experimental map of the residual total magnetic field (fig. 3a) and the synthetic map (fig. 3b) obtained assuming a magnetic dipole centred at $x=12 \mathrm{~km}, y=17 \mathrm{~km}$ and $z=-2 \mathrm{~km}$, with magnetic moment of $110 \mathrm{nT}$, inclination $56.30^{\circ}$ and declination $-1^{\circ}$. The misfit between the two maps is in modulus less than $2 \%$. Therefore, the synthetic dipole can represent a simple model of the observed residual magnetic anomaly field in the Vesuvius volcanic area. Concluding, this leads to hypothesize the existence of a uniformly magnetised material filling the top terminal portion of the volcano, exactly where gravity data indicate a low density (Iuliano et al., 2001). 


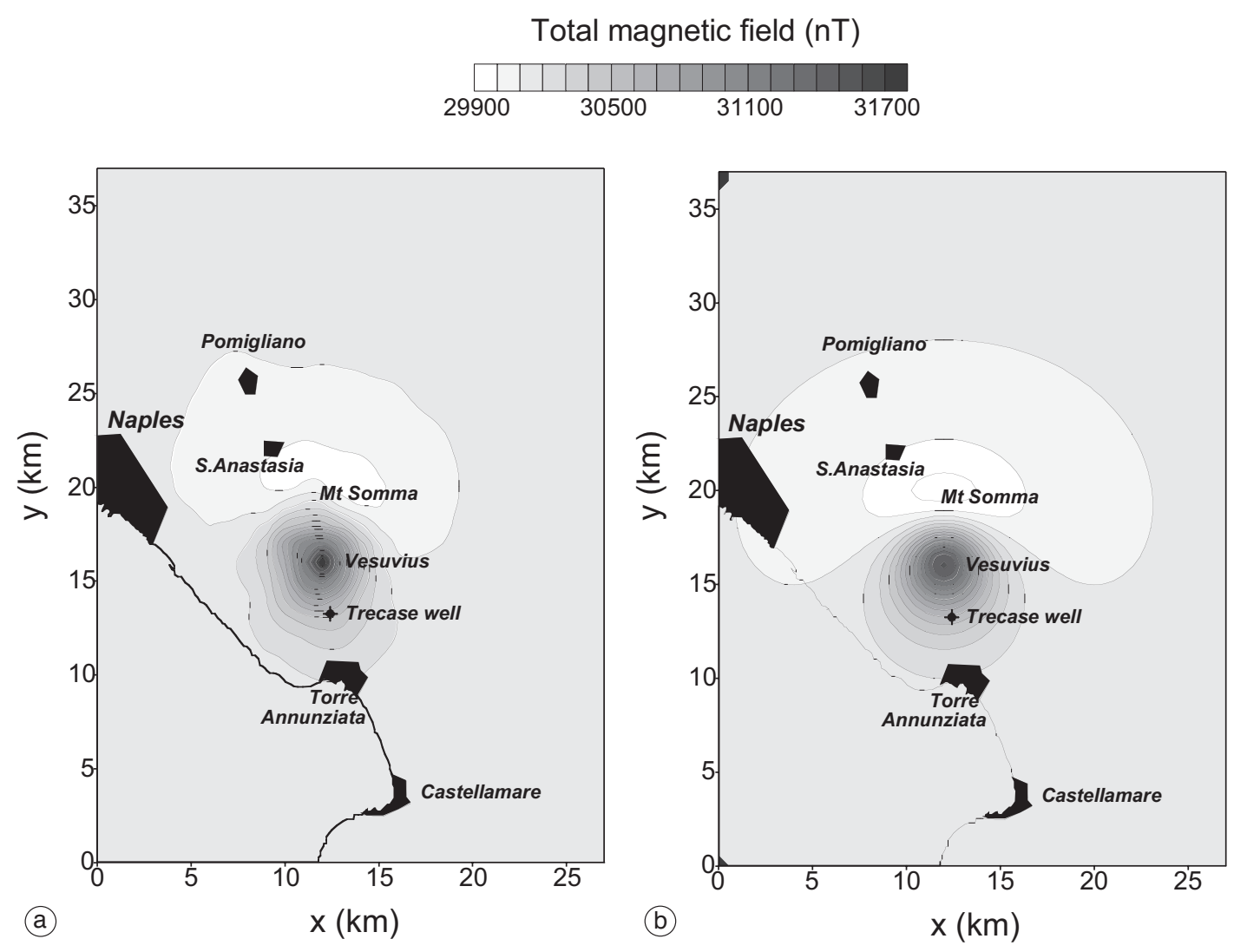

Fig. 3a,b. Comparison between the observed map of the residual total magnetic field (a) and the synthetic map (b) obtained assuming a magnetic dipole centred at $x=12 \mathrm{~km}, y=17 \mathrm{~km}$ and $z=-2 \mathrm{~km}$, with magnetic moment $110 \mathrm{nT}$, inclination $56.30^{\circ}$ and declination $-1^{\circ}$.

\section{REFERENCES}

AdVANCED VisuAL SySTEMS InC. (1998): AVS/Express manual. Waltham, MA, USA.

BLAKELY, R.J. (1996): Potential Theory in Gravity and Magnetic Applications (Cambridge University Press), pp. 461.

CASSANO, E. and P. LA TORRE (1987): Geophysics, in: Somma Vesuvius, edited by R. SANTACROCE, Quad. Ric. Sci., 114/8, 175-196, Consiglio Nazionale delle Ricerche, Rome.

Di Maio, R., P. Mauriello, D. Patella, Z. Petrillo, S. Piscitelli and A. SinisCAlCHI (1998): Electric and electromagnetic outline of the Mount Somma-Vesuvius structural setting, J. Volcanol. Geotherm. Res., 82, 219-238
IUliano, T., P. MAURIELlo and D. PATElla (2001): Looking inside Mount Vesuvius by potential fields integrated probability tomographies, J. Volcanol. Geotherm. Res. (in press).

JACKSON, J.D. (1998): Classical Electrodynamics (J. Wiley and Sons, New York), pp. 832.

Mauriello, P. and D. PAtella (1999a): Resistivity anomaly imaging by probability tomography, Geophys. Prospect., 47, 411-429.

Mauriello, P. and D. Patella (1999b): Principles of probability tomography for natural-source electromagnetic induction fields, Geophysics, 64, 1403-1417.

Mauriello, P. and D. PATElla (2000): A physical pattern recognition approach for 2D electromagnetic induction studies. Ann. Geofis., 43 (2), 343-359.

MAURIELlO, P. and D. PATELLA (2001a): Gravity probability 
tomography: a new tool for buried mass distribution imaging, Geophys. Prospect., 49, 1-12.

Mauriello, P. and D. PATElla (2001b): Localization of maximum-depth gravity anomaly sources by a distribution of equivalent point masses, Geophysics, 66, 1431-1437.

Mauriello, P. and D. Patella (2001c): Magnetic buried sources discrimination by probability tomography, Geophys. Prospect. (submitted).

Mauriello, P., D. Monna and D. Patella (1998): 3D geoelectric tomography and archaeological applications, Geophys. Prospect., 46, 543-570.
PARASNIS, D.S. (1997): Principles of Applied Geophysics (Chapman \& Hall, London), pp. 429.

PATElla, D. (1997a): Introduction to ground surface selfpotential tomography, Geophys. Prospect., 45, 653-681.

PATElla, D. (1997b): Self-potential global tomography including topographic effects, Geophys. Prospect., 45, 843-863.

PATElla, D. and P. MAuriello (1999): The geophysical contribution to the safeguard of historical sites in active volcanic areas. The Vesuvius case-history, J. Appl. Geophys., 41, 241-258. 